\title{
The Flash Stimulated VEP in the Diagnosis of Glaucoma
}

\author{
M. T. WATTS, P. A. GOOD, E. C. O'NEILL \\ Birmingham
}

\begin{abstract}
Summary
The Visual Evoked Potential using pattern stimulation has been used in recent years as an attempt to make an early diagnosis of glaucoma. However because of the macular dominance of responses obtained by this method, only diseases involving the papillomacular bundle reveal abnormality; such conditions do not occur in glaucoma. In this study we report the use of the early component (P1) of the flash stimulated visual evoked potential in the diagnosis of primary open angle glaucoma.

It appears to provide an objective, accurate and repeatable screening technique. The size of P1 correlates well with the size of optic disc cupping, and field loss. The relationships between systemic vascular disease, $P 1$ reductions, and glaucoma, lend support to the ischaemic origins of the disease.
\end{abstract}

A number of techniques have been developed to diagnose and monitor primary open angle glaucoma (POAG). ${ }^{1}$ The search for ways of making an earlier diagnosis, and hence improving the prognosis of the disease ${ }^{2}$ continues, with varying degrees of success. The three parameters which have been most widely used are the appearance of the optic nerve head, the intraocular pressure, and distribution of any field loss.

It has been shown that up to $40 \%$ of the total nerve fibres at the nerve head may be lost before any significant changes are found on perimetry, ${ }^{3}$ and similarly, pathological cupping represents advanced nerve damage. To detect POAG at an early stage, a number of advanced automated perimetric techniques have been developed, ${ }^{4}$ however they are limited by their subjectivity, particularly in elderly patients.

The use of pattern ERGs, measurements of contrast sensitivity, ${ }^{2}$ and photographic assess- ment of the optic disc, ${ }^{5}$ have been claimed to be successful in the early diagnosis of glaucoma.

In recent years studies of visually evoked potentials (VEP) have been used in order to identify and monitor nerve fibre damage ${ }^{6-10}$ All these have concentrated on using checkerboard pattern stimulation with measurement of the P100, or major, component of the VEP. However since this component is generated from within the papillomacular bundle region of the retina, ${ }^{11}$ it may not be affected until very late in the course of glaucomatous nerve damage, which preferentially affects nerve fibres serving the peripheral retina. This limits the usefulness of pattern VEP in the diagnosis of glaucoma considerably, ${ }^{12}$ particularly since it is also dependent on good optical refraction, clear media, and patient cooperation. These latter factors are particularly relevant in an elderly population, who are most likely to suffer glaucoma.

From: Retina Research Department, Birmingham \& Midland Eye Hospital.

Correspondence to: Mr M. T. Watts, Ophthalmic Department, Royal Hallamshire Hospital, Glossop Road, Sheffield. 
We have previously shown ${ }^{13}$ that the $\mathrm{P} 1$, or early, component of the flash stimulated VEP is reduced in POAG, compared to normal eyes. In the present study an assessment is made of the use of the flash evoked P1 component, in the early diagnosis of POAG, by comparing it with the conventional criteria of optic nerve head appearance, intraocular pressure, and field loss.

\section{Materials and methods}

Seventy-four patients (130 eyes), diagnosed as suffering from POAG, were selected from patients attending the glaucoma clinic at the Birmingham and Midland Eye Hospital between May 1987 and February 1988, for study of optic nerve function by flash evoked VEP analysis. Informed consent was obtained to perform electrodiagnosis. The mean age of the patients was 66 years, ranging from 38-86 years. All had been diagnosed as suffering from POAG by fulfilling the criteria of one of the following groups:

1. Typical arcuate glaucomatous field loss in the presence of ocular hypertension, with or without nerve head appearances suggestive of glaucoma.

2. Ocular hypertension in association with nerve head appearances suggestive of glaucoma, but with either normal visual fields, or only a small nasal step in the field, or enlarged blindspot.

Patients were excluded from this study if they had advanced glaucoma, defined as greater than $50 \%$ field loss, or had ocular hypertension alone, or had previously been diagnosed as having venous/arterial occlusion or other retinal pathology such as retinal detachment or myopic degeneration.

The study therefore consisted of 56 patients with bilateral POAG, and 18 patients with unilateral POAG.

A control group of 125 patients (250 eyes), mean age 68 years, age range 35-90 years, was also selected from patients referred for VEP assessment who were subsequently found to have no ocular abnormality other than unilateral lens opacities (53 eyes). These 53 eyes were used to assess the effect of opaque media on the P1 component of the VEP, since 26 of the patients with POAG also had lens opacity.
The VEPs were recorded using a Nicolet C4 clinical averager. Scalp electrodes were placed according to the international EEG montage convention (10/20) with the active electrode being placed on FZ, and the reference electrode on OZ. All positive waves therefore appeared in a downward direction.

The flash stimulus was provided by a Grass PS 22 photostimulator. The stimulus intensity was presented at intensity setting 2 (1500 LUX), with the exception of the patients with lens opacity, who were presented with a flash stimulus set at intensity 8 (6200 LUX). The VEP was recorded over a time base of 200 msec., the low pass filter being set at $100 \mathrm{~Hz}$, and the high pass filter at $1 \mathrm{~Hz}$. Forty averages were obtained from each eye separately, the stimulus rate being $1.5 \mathrm{~Hz}$.

The Visual Fields were recorded using either a Goldmann perimeter or Octopus automated perimeter. The area of field loss, if present, was measured, and the visual fields classified as: (a) no field loss, (b) abnormal blind spot, (c) $<15 \%$ field loss, (d) $>15 \%$ field loss.

Statistical analysis of the data tabulated in Tables 1 and 2 was obtained using the pooled $t$ test.

\section{Results}

Table I shows the values of $\mathrm{P} 1$ and $\mathrm{P} 2$ measured in the group of patients with POAG compared to the control group of 'normal' eyes. This shows a significant reduction of P1 in the POAG group but no reduction of $\mathrm{P} 2$.

Comparing the value of $\mathrm{P} 1$ with the degree of field loss demonstrates some correlation between the severity of field loss and the reduction of P1 (Fig. 1).

The good correlation between $\mathrm{P} 1$ reductions in the POAG group, and diagnosis made by conventional criteria, is further highlighted by comparing the P1 amplitudes of the affected and 'normal' eyes in the 18 patients with unilateral POAG (see Table II). Once again the significant reduction in P1 amplitude in the affected eye was not mirrored by a similar reduction in P2 (see Fig. 2).

One of the potential uses of P1 measurement is in eyes where the optic disc is not visible. It is therefore interesting to note that, 
Table I. POAG versus normal control.

\begin{tabular}{lcccccccc}
\hline & \multicolumn{3}{c}{ POAG } & & \multicolumn{3}{c}{ Control } & \\
\cline { 2 - 3 } Component & $n$ & mean & s.d. & & $n$ & mean & s.d. & Significance \\
\hline P1 (uV) & 130 & 1.83 & 1.70 & & 250 & 6.56 & 6.20 & p $<.001$ \\
P2 (uV) & 103 & 6.85 & 6.44 & & 250 & 7.39 & 6.98 & N.S. \\
\hline
\end{tabular}

Table II. Unilateral POAG; affected versus normal eye.

\begin{tabular}{|c|c|c|c|c|c|c|c|}
\hline \multirow[b]{2}{*}{ Component } & \multicolumn{3}{|c|}{ Affected eye } & \multicolumn{3}{|c|}{ Normal eye } & \multirow[b]{2}{*}{ Significance } \\
\hline & $n$ & mean & s.d. & $n$ & mean & s.d. & \\
\hline P1 (uV) & 18 & 1.61 & 1.46 & 18 & 4.56 & 2.20 & $\mathrm{p}<.005$ \\
\hline P2 (uV) & 18 & 6.62 & 5.95 & 18 & 7.20 & 5.82 & N.S. \\
\hline
\end{tabular}

in those eyes in which an accurate assessment of the optic nerve head could be made, the reduction in $\mathrm{P} 1$ amplitude correlated well with the ratio of vertical cup to disc diameter (Fig. 3). This relationship shows a Pearson's correlation coefficient $(\gamma)$ of 0.9986 , which gives a significance of $\mathrm{P}<0.05$.

Using data from the 53 patients in the control group with unilateral cataract, the effect of opaque media on the P1 and P2 components was assessed by comparing the eye with opaque media with that having clear media. The results shown in Table III reveal no significant difference in either the $\mathrm{P} 1$ or $\mathrm{P} 2$ components.

The amplitude of P1 is affected by age ${ }^{14}$ and systemic vascular disease (hypertension, or history of cerebrovascular accident, or myo-

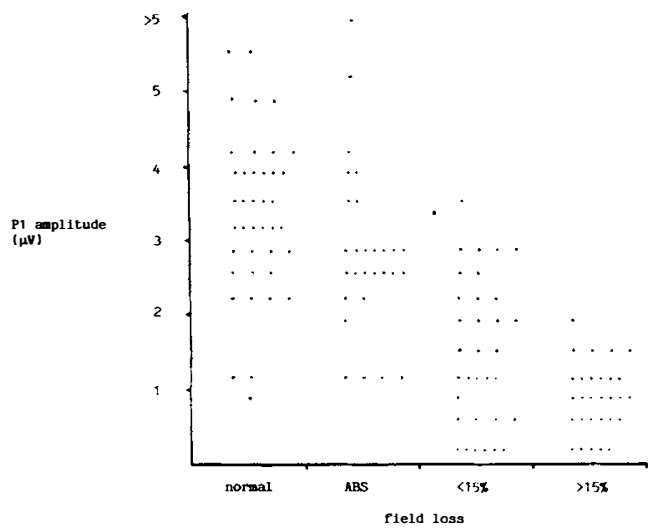

Fig. 1 Scatter plot of P1 amplitude versus field loss, revealing a correlation between $P 1$ reduction and degree of field loss. $(A B S=$ abnormal blind spot $)$. cardial infarct/angina pectoris $)^{13}$. In this study 28 of the 74 patients with POAG had evidence of systemic vasclar disease. In this sub-group the mean $\mathrm{P} 1$ value was $1.74 \mathrm{uV}(\mathrm{SD} \pm 0.83$ $\mathrm{uV}$ ) as compared to a mean PI value of 2.92 $\mathrm{uV}(\mathrm{SD} \pm 1.21 \mathrm{uV})$ in the remaining 46 patients with POAG but no overt evidence of systemic vascular disease. The difference between these groups was highly significant: $\mathrm{p}<0.005$. On comparing the $\mathrm{P} 2$ values in the same groups the respective values were 6.78 $\mathrm{uV}(\mathrm{SD} \pm 6.67)$, and 6.92 uV $(\mathrm{SD} \pm 5.88 \mathrm{uV})$ there being no significant difference in P2 values.

In order to compensate for the tendency of P1 normally to increase with age, a normal value of $=>4 \mathrm{uV}$ was chosen for patients $<75$ years old, and $=>5 \mathrm{uV}$ for patients $=>75$ years old. Using these normal values $93 \%$ of the patients identified as having POAG by conventional diagnostic criteria had a reduc-
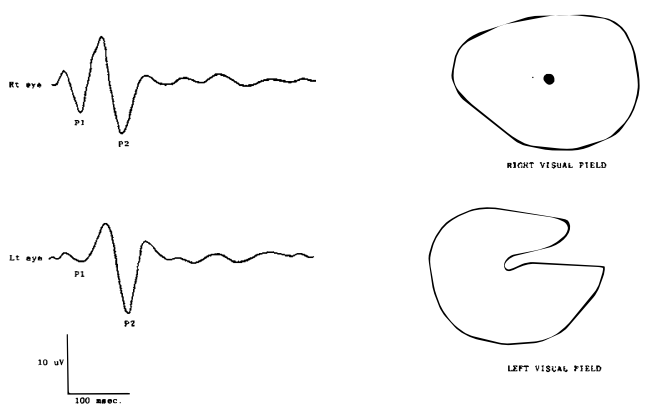

Fig. 2 Patient $M F$ aged 61, unilateral POAG (left eye). Note the loss of $P 1$ and preservation of the P2 component. 


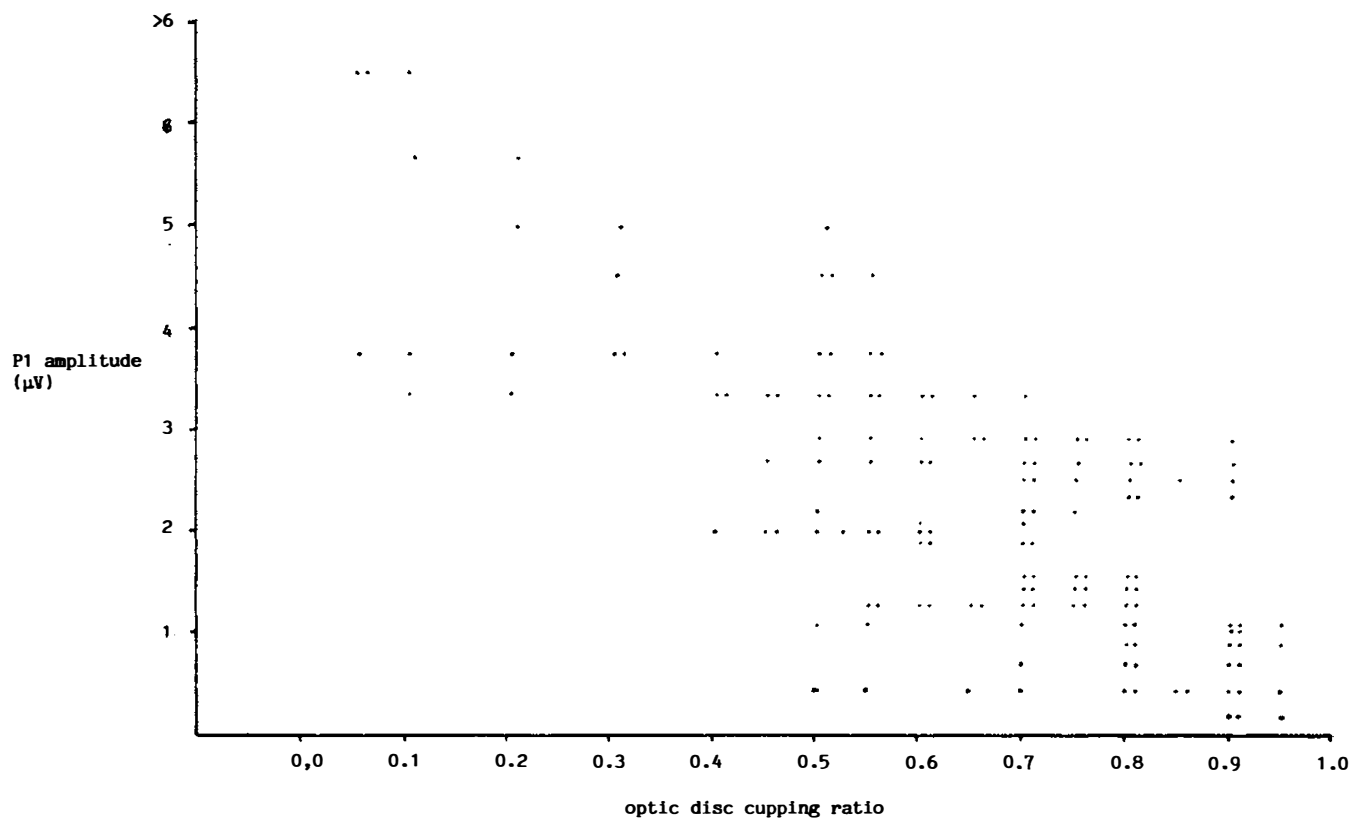

Fig. 3 Scatter plot of P1 amplitude versus optic disc cupping ratios, showing a correlation between reductions of $P 1$ and increasing cup size.

tion of P1 (see Fig. 4). In the control group these normal values produced a false positive (i.e. reduced P1) of $20 \%$.

\section{Discussion}

Correlation of a reduced $\mathrm{P} 1$ with presence of POAG, as defined by conventional criteria, was good, and as such it appears that this technique has potential as a screening method. The test is quick to perform (five minutes) and requires little patient cooperation. By using flash instead of pattern stimulation the problems of opaque media, incorrect refraction, pupillary anomalies and stimulus presentation are minimized.

Analysis of results requires little expertise, and can be readily compared with previous results, as part of an on-going monitoring pro- cedure, which would be of possible value in glaucoma suspects and ocular hypertensives. The results have a high degree of specificity and sensitivity. ${ }^{13}$

The correlation between the size of the optic cup and reduction of $\mathrm{P} 1$ is significant, and this suggests that the origins of P1 closely relate to nerve fibre losses in this area. Where cupping of the disc is equivocal, or the nerve head not visible, the presence of a reduced P1 may provide sufficient evidence for a diagnosis of POAG to be made.

A significant relationship exists between very low $\mathrm{P} 1$ values and systemic vascular disease $(p<0.005)$. This finding combined with evidence of a reduced $\mathrm{P} 1$ in patients with systemic vascular disease but no evidence of POAG, as found in our previous study, ${ }^{14}$ lends support to those authors ${ }^{15-17}$ who have sug-

Table III. Affect of Opaque media on P1 and P2.

\begin{tabular}{lccccccccc}
\hline & \multicolumn{3}{c}{ Opaque media } & & \multicolumn{3}{c}{ Clear media } & \\
\cline { 2 - 4 } \cline { 7 - 8 } Component & $n$ & mean & s.d. & & $n$ & mean & s.d. & Significance \\
\hline P1 (uV) & 53 & 6.95 & 6.25 & & 53 & 6.62 & 6.03 & N.S. \\
P2 (uV) & 53 & 7.81 & 7.01 & & 53 & 7.43 & 6.82 & N.S. \\
\hline
\end{tabular}



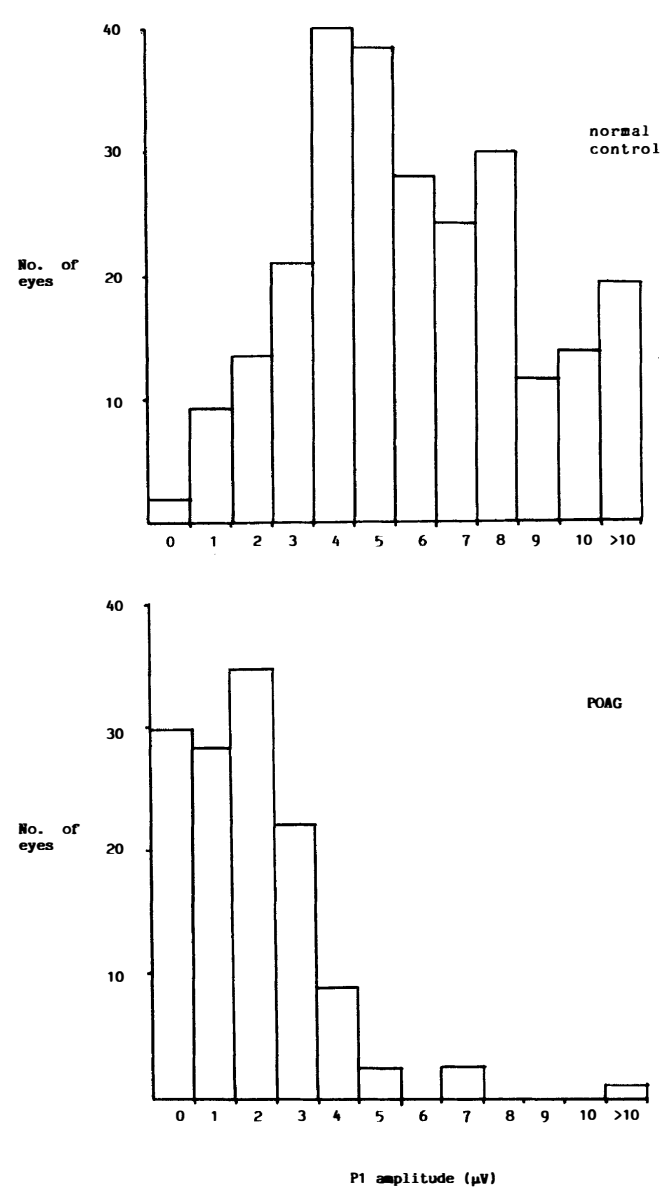

Fig. 4 Distribution of P1 values in the 'normal' control group, compared with the POAG group.

gested that glaucomatous nerve fibre damage is primarily a vascular process. However the reduction of $P 1$ in systemic vascular disease, without POAG, appears to produce a relatively high number of false positives, and therefore this means that a reduction in P1 must be interpreted with caution in arteriopaths.

In summary it appears that the value of the P1 component correlates well with conventional criteria used in the diagnosis and monitoring of POAG. Its ease of measurement, combined with its total objectivity, offer a potentially useful screening technique, as well as an index of the progression of the disease.
In cases of doubt evaluation of the P1 component may be of value in either making or refuting a diagnosis of POAG, particularly where field loss is absent, equivocal or may relate to other diseaseprocesses.

Further studies may reveal whether preservation of P1 during the progression of the disease has prognostic indications, and could help in decisions regarding the management of glaucoma.

We should like to thank Miss K. Levell and Mr. J. McLaughlin for technical assistance, and Mr. N. Galloway FRCS, for reading the manuscript.

\section{References}

${ }^{1}$ Spaeth GL and Varma R: Assessment of the glaucomatous patient. Eye 1987, 1: 29-39.

${ }^{2}$ Smith RJH: The enigma of primary open angle glaucoma. Trans Ophthalmol Soc UK 1986, 105: 618-29.

${ }^{3}$ Quigley HA, Addicks EM, Green WR: Optic nerve damage in human glaucoma III. Quantitative correlation of nerve fibre loss and visual field defect in glaucoma, ischaemic neuropathy, disc oedema, and toxic neuropathy. Arch Ophthalmol 1982, 100: $135-46$.

${ }^{4}$ Sommer A, Enger C, Witt K: Screening for glaucomatous visual field loss with automated threshold perimetry. Am J Ophthalmol 1987, 103: 681-4.

${ }^{5}$ Klein BEK, Moss SE, Magli YL, Klein R, et al. Optic disc cupping as clinically estimated from photographs. Ophthalmology 1987, 94: 1481-6.

${ }^{6}$ Howe JW and Mitchell KW: Visual evoked potential changes in chronic glaucoma and ocular hypertension. Trans Ophthalmol Soc UK 1986, 105: 457-62.

${ }^{7}$ Towle VL, Moskowitz A, Sokol S, Schwartz B: The visual evoked potential in glaucoma and ocular hypertension. Effects of check size, field size and stimulation rate. Invest Ophthalmol Vis Sci 1983, 24: $175-83$.

${ }^{8}$ Cappin JM and Nissim S: Visual evoked responses in the assessment of field defects in glaucoma. Arch Ophthalmol 1975, 93: 9-18.

${ }^{9}$ Sokol S, Domar A, Moskowitz A, Schwartz B: Pattern evoked potential latency and contrast sensitivity in glaucoma and ocular hypertension. Doc Ophthalmol Proc Serv 1981, 27: 79-86.

${ }^{10}$ Galloway NR and Barber C: the transient pattern onset VEP in glaucoma. Doc Ophthalmol Proc Serv 1981, 27: 95-101.

${ }^{11}$ Ikeda $\mathrm{H}$ and Friedmann AI: Electrophysiology of the retina and visual pathway. In: Modern Oph- 
thalmology, Sorsby A, (ed.) London, Butterworth, 1972, 38-55.

${ }^{12}$ Galloway NR: Evoked responses and the eye. Trans Ophthalmol Soc UK 1986, 105: 273-86.

${ }^{13}$ Good PA, Marsters JB, Mortimer MJ: Flash stimulation evoked potentials in the diagnosis of chronic glaucoma. Lancet 1987, 1: 1259-60.

${ }^{14}$ Wright CE, Williams DE, Drasdo N, Harding GFA: The influence of age on the electroretinogram and visual evoked potential. Doc Ophthalmol 1985, 59: $365-70$.
${ }^{15}$ Quigley HA: Reappraisal of the mechanisms of glaucomatous optic nerve damage. Eye 1987, 1: 318-22.

${ }^{16}$ Drance SM: Is ischaemic the villain in glaucomatous cuping and optic atrophy. In: Controversy in Ophthalmology. Brockhurst FJ, Boruchoff SA, Hutchinson BI and Lessel S, (eds.) Philadelphia, Saunders WB, 1977, 292-300.

${ }^{17}$ Harrington DO: Glaucoma. In: The Visual Fields: A textbook and atlas of clinical perimetry. St Louis, Mosby CV 1981, 176-212. 